\title{
Hemodynamic Disturbances in Premature Infants Born after Chorioamnionitis: Association with Cord Blood Cytokine Concentrations
}

\author{
TOBY DEBRA YANOWITZ, JEANNE ANN JORDAN, CAROL HUNTRESS GILMOUR, \\ RICHARD TOWBIN, A'DELBERT BOWEN, JAMES MICHAEL ROBERTS, AND \\ BEVERLY SOBCHAK BROZANSKI
}

Department of Pediatrics, Division of Neonatology [T.D.Y., C.H.G., B.S.B.], Department of Pathology [J.A.J.], Department of Radiology [R.T., A.B.], Department of Obstetrics, Gynecology and Reproductive Sciences [J.M.R.], University of Pittsburgh School of Medicine and the Magee-Womens Research Institute, Pittsburgh, PA, U.S.A.

\begin{abstract}
Chorioamnionitis and elevated cord blood inflammatory cytokine concentrations are associated with detectable disturbances of systemic and cerebral hemodynamics in premature newborns. Fifty-five infants (25-31 wk gestation) were enrolled. Chorioamnionitis was defined by placental histology. IL-6, IL-1 $\beta$, and tumor necrosis factor- $\alpha$ were quantified by ELISA. Blood pressure, heart rate, cardiac output, stroke volume, fractional shortening, and middle cerebral artery blood flow velocities were measured at $3 \pm 1 \mathrm{~h}$ after birth. Chorioamnionitis was evident in 22 placentas and was associated with increased IL-6 $(p<0.001)$, IL-1 $\beta(p=0.035)$, and heart rate $(p=0.027)$; and with decreased mean and diastolic blood pressure $(p=0.026$ and $p=$ 0.019 , respectively). IL-6 concentration correlated inversely with systolic, mean, and diastolic blood pressures. Right ventricular cardiac output was elevated $(p=0.028)$ in infants with fetal vessel inflammation. Maternal temperature $\geq 38.0^{\circ} \mathrm{C}$ and newborn immature-to-total white blood cell ratio $\geq 0.4$ were associated with significant decreases in left ventricular fractional shortening ( $p=0.001$ and $p=0.005$, respectively). Neither chorioamnionitis nor elevated cytokine concentrations were as-
\end{abstract}

\section{ABSTRACT}

sociated with changes in middle cerebral artery Doppler blood flow velocities. Chorioamnionitis and elevated cord blood IL-6 concentrations are associated with decreased blood pressure in premature newborns. Inflammation of the fetal vessels and nonspecific indicators of infection are associated with disturbances in cardiac function. Infants with chorioamnionitis and elevated cytokine concentrations do not manifest changes in cerebral Doppler indices within the first few postnatal hours. We speculate that cytokine-associated systemic hemodynamic disturbances in premature infants born after chorioamnionitis predispose such infants to perinatal brain injury. (Pediatr Res 51: 310-316, 2002)

\section{Abbreviations}

TNF- $\alpha$, tumor necrosis factor- $\alpha$

PVL, periventricular leukomalacia

IVH, intraventricular hemorrhage

IL-6, interleukin-6

IL-Ib, interleukin-1-beta
Hemodynamic abnormalities among premature infants are recognized to be important factors in the pathogenesis of perinatal brain injury. For example, IVH is more common in very low birth weight infants with hypotension (1) and fluctuating cerebral blood flow velocities (2). PVL may denote white matter ischemia (3) that results from compromised cerebral blood flow during a critical developmental period in which oligodendroglia undergo myelogenesis (4) in the presence of a tenuous vascular supply $(5,6)$. In fact, animals at a stage of

Received February 28, 2001; accepted November 12, 2001.

Correspondence and reprint requests: Toby Debra Yanowitz, MD, Department of Pediatrics, Magee-Womens Hospital, 300 Halket Street, Pittsburgh, PA 15213, U.S.A.; e-mail: tyanowitz@mail.magee.edu

Supported by the American Heart Association (grant 9806419U) and the National Institutes of Health (grant K23 HD01317), and the 25 Club of Magee-Womens Hospital. brain development comparable to the $<32$-wk human fetus display a distinct and selective reduction of white matter blood flow in response to hypotension (7), confirming the predisposition for periventricular white matter ischemia at this gestational age.

The ischemia theory of white matter injury has been challenged $(8-11)$ by the epidemiologic observation that chorioamnionitis is a strong predictor of subsequent $\operatorname{PVL}(12,13)$ and cerebral palsy in both term (14) and preterm (13) infants. Although the mechanism of white matter injury in the context of infection has not been fully elucidated, evidence suggests that the proinflammatory cytokines are involved. IL-6, IL-1 $\beta$, and TNF- $\alpha$ concentrations are elevated in both amniotic fluid (15) and cord blood (16) of pregnancies complicated by cho- 
rioamnionitis, and are associated with multiple organ system morbidities among premature infants $(17,18)$. The link between IL-6 and PVL is particularly strong $(11,13)$.

Cytokines have toxic effects (19) on immature oligodendroglia in vitro that closely resemble, and may explain, the pathologic findings of PVL (20). If, however, white matter damage results from a hypoxic-ischemic insult in the premature brain, then the known in vivo hemodynamic effects of cytokines may explain their association with PVL. In experimental animals, intravascular cytokine injection produces systemic hypotension $(19,20)$ and exerts species-specific effects on cerebrovascular tone $(21,22)$, potentially altering cerebral blood flow. Furthermore, cytokines can disrupt the blood-brain barrier $(23,24)$ and produce cerebral edema $(25)$, thus decreasing cerebral blood flow. The effects of cytokines on the human premature newborn's systemic and cerebral circulations have not been investigated previously.

The present study was designed to explore the association between chorioamnionitis, proinflammatory cytokines, nonspecific markers of inflammation, and hemodynamic function in premature newborns at risk for brain injury. We hypothesized that chorioamnionitis and increased concentrations of the proinflammatory cytokines would be associated with detectable disturbances of systemic hemodynamics and cerebral perfusion, and would thereby suggest a possible mechanism for cytokine-associated brain injury in premature infants.

\section{METHODS}

This study was approved by the Institutional Review Board of Magee-Womens Hospital.

Subject enrollment and standard care. Informed consent was obtained prenatally from mothers projected to be delivered at $<32$ wk gestation between April 1, 1998, and October 30, 1999. Gestational age assessment was based on either a first trimester ultrasound or the last menstrual period if no early ultrasound was available. Exclusion criteria included congenital anomalies of the brain, heart, or kidneys; intrauterine growth restriction [estimated fetal weight below the third percentile for gestational age, no growth on three serial prenatal ultrasounds, or birth weight $<2$ SD below the mean (26)]; in utero demise of a twin; twin-to-twin transfusion sequence; delivery after $32 \mathrm{wk}$ gestation; and inability to obtain informed consent before delivery. Fifty-five newborns (45 singleton and 10 twin infants) were enrolled.

Maternal-fetal pairs and newborn infants were cared for by physicians not involved in the hemodynamic studies. The use of tocolytic agents, antenatal betamethasone, antibiotics, fluid resuscitation, vasopressors, ventilation, and surfactant administration were at the discretion of the caregivers. No infant received postnatal glucocorticoid or indomethacin before hemodynamic evaluation. Clinical diagnoses and interventions, baseline demographic findings, blood gas values, blood culture results, and nonspecific markers of infection, including maternal and newborn white blood cell counts, maternal temperature, and newborn immature-total white blood cell ratio, were recorded from the medical record after the hemodynamic study was completed.
Cytokine assays. After delivery of the placenta, $1-5 \mathrm{~mL}$ of cord blood was collected in a Vacutainer tube (BD, Franklin Lakes, NJ) containing the anticoagulant EDTA. The sample was centrifuged at $3000 \mathrm{~g}$ for $10 \mathrm{~min}$ to separate the plasma. Within $1 \mathrm{~h}$ of collection, plasma samples were labeled with a coded subject number and stored at $-80^{\circ} \mathrm{C}$ in several $0.5-\mathrm{mL}$ aliquots. Samples did not undergo freeze-thaw cycles, and were stored for up to 12 mo before cytokine testing occurred in two batches. IL- 6 , IL- $1 \beta$, and TNF- $\alpha$ concentrations were measured (J.J.) by ELISA (R\&D Systems, Minneapolis, MN, U.S.A.). Each cytokine sample was run in duplicate, and the mean cytokine concentration calculated. Assay sensitivities were $<0.07 \mathrm{pg} / \mathrm{mL}$ for IL- $6,<1.0 \mathrm{pg} / \mathrm{mL}$ for IL- $1 \beta$, and 4.4 $\mathrm{pg} / \mathrm{mL}$ for TNF- $\alpha$. The published (R\&D Systems) intra- and interassay coefficients of variation averaged, respectively, 2.6 and 4.5 for IL-6, 4.8 and 5.6 for IL- $1 \beta$, and 4.7 and 5.8 for TNF- $\alpha$.

Definition of chorioamnionitis. Chorioamnionitis was defined by pathologic criteria because infection has been identified frequently by placental histology in women who deliver preterm without clinical signs of chorioamnionitis $(15,27)$. Chorioamnionitis was diagnosed when $>10$ neutrophils per high-power field were present in the chorion or amnion of routine hematoxylin and eosin stained sections of membrane rolls and umbilical cords. Chorioamnionitis was further categorized by the presence or absence of fetal vessel inflammation, defined as fetal vasculitis and/or funisitis. Fetal vasculitis was diagnosed by infiltration of neutrophils into the fetal chorionic stem vessels, and funisitis by the infiltration of neutrophils into the umbilical vessels and cord.

Hemodynamic examination. Hemodynamic evaluations were performed when infants were $2-4 \mathrm{~h}$ old. Infants were examined supine on an open warmer in the neonatal intensive care unit with the ultrasound gel warmed to near body temperature. Because not all infants had indwelling arterial catheters and oscillometric mean blood pressure has been shown to correlate well with intra-arterial mean blood pressure (26), oscillometric blood pressure (Dynamap, Critikon, Tampa, FL, U.S.A.) was measured for all infants. Inspired oxygen concentration was adjusted to maintain pulse-oximeter saturations between $92 \%$ and $98 \%$ during the examination.

Hemodynamic studies were performed on a Hewlett Packard SONOS 2000 echocardiograph (Andover, MA, U.S.A.) using the $7.5-\mathrm{mHz}$ transducer for tissue imaging and $5.0 \mathrm{mHz}$ for Doppler recording. Measurements were made in the following order: left and then right middle cerebral artery blood flow velocities, left and then right ventricular cardiac outputs, stroke volume, left ventricular fractional shortening, and ductus arteriosus patency. Heart rate and blood pressure were recorded immediately after each cerebral vessel Doppler recording. All studies were performed by a single investigator (T.Y.) who was unaware of the maternal and infant clinical histories. The intraobserver variability was $2.6 \%$ for mean cerebral blood flow velocity, $5.8 \%$ for peak systolic blood flow velocity, $8.0 \%$ for cardiac output, and $9.8 \%$ for fractional shortening.

Middle cerebral artery measurements were made just distal to the origin of the artery from the circle of Willis, with the transducer perpendicular to the pterion of the temporal bone. 
Color flow mapping was used to identify the artery and pulsed wave Doppler applied to determine blood flow velocity. Peak systolic, mean, and end-diastolic blood flow velocity measurements were each averaged over five consecutive waves in the area of highest velocity. Relative vascular resistance was estimated by mean arterial blood pressure divided by mean blood flow velocity (28).

Left and right ventricular cardiac outputs were measured from the suprasternal notch and a left parasternal short axis view, respectively, with the flow volume sampled in the middle of the outflow tract at the level of the valve leaflet insertion. Velocity waveforms were considered optimal when the leaflet signal was visible on both sides of the waveform and the characteristic sound of the Doppler signal was loudest. The internal diameter of the valve annulus was measured from the tissue image at the point of maximal valve leaflet separation. Average cardiac output (milliliters per minute) was calculated from five cardiac cycles. Left ventricular fractional shortening was determined by M-mode from the left parasternal short axis view at a level just below the mitral valve. Ductus arteriosus patency and direction of flow were determined by color flow mapping and confirmed by continuous wave Doppler.

Cranial sonography. Cranial ultrasounds were performed within $72 \mathrm{~h}$ of birth and again on d 14 by a clinical technician using an Acuson Sequoia (Acuson, Mountain View, CA, U.S.A.) with an $8.0-\mathrm{mHz}$ transducer. All studies were interpreted by two radiologists (R.T. and A.B.) unaware of the maternal and neonatal histories, cardiac functions, cerebral blood flow velocities, and cytokine concentrations. Cranial ultrasounds were scored as bilateral/left/right IVH [Papile grade (29)], cystic PVL, periventricular hyperechogenicity, ventriculomegaly, or normal.

Statistical analysis. Sample size was calculated to enable detection of a $75 \mathrm{~mL} / \mathrm{min}$ difference in cardiac output, a $5 \%$ difference in fractional shortening, or a $5 \mathrm{~cm} / \mathrm{s}$ difference in mean middle cerebral artery blood flow velocity between infants with and without chorioamnionitis with $90 \%$ power and an $\alpha$ error of 0.05 . Hemodynamic measures were assumed to be normally distributed and continuous. Unpaired $t$ test was used to analyze the effects of chorioamnionitis on hemodynamic variables. The nonparametric Kruskal-Wallis test was used to assess differences where the group sizes were unbalanced. ANOVA with Tukey's post hoc test was used when comparisons among three groups were made. Fisher's exact test was used for comparisons of dichotomous variables. Univariate relationships between continuous variables were assessed by Pearson's correlation. Multiple linear or logistic regression was used to determine predictors of blood pressure, $\mathrm{IVH}$, and white matter injury, as appropriate.

Concentrations of IL- 6, IL- $1 \beta$, and TNF- $\alpha$ were expected to follow a non-normal distribution. Logarithmic transformation was used to normalize cytokine concentrations before parametric analysis. Cytokine data were dichotomized as detectable versus nondetectable for categorical analyses. In categorical analyses of maternal fever and the newborn immature-to-total white blood cell count (I/T ratio), cut-off points of $38^{\circ} \mathrm{C}$ and 0.4 , respectively, were chosen a priori to identify the mothers and infants with the highest degrees of systemic inflammatory response. Statistical analyses were completed using MINITAB for Windows, Release 12.23 (Minitab, State College, PA, U.S.A.) and Stata 6.0 (Stata, College Station, TX, U.S.A.).

\section{RESULTS}

\section{Clinical Features of the Study Population}

Twenty-two infants had histologic evidence of chorioamnionitis. Eleven of these had fetal vessel inflammation. No placenta had fetal vessel inflammation in the absence of chorioamnionitis. Infants with and without chorioamnionitis had similar demographic characteristics (Table 1), Apgar scores (median at 1 and 5 min: 7 and 8 versus 6 and 9, respectively, for infants with chorioamnionitis versus without), antenatal steroid exposure [median (range): 2 (1-6) doses for each group], incidence of respiratory distress syndrome (50\% versus $55 \%$ ), need for respiratory support, and blood gas values (Table 1).

Risk factors for and laboratory signs of infection are shown in Table 2. As indicated in Table 2, the clinical diagnosis of chorioamnionitis was made infrequently, and at similar rates for mothers with and without pathologically confirmed chorioamnionitis. Mothers with histologic evidence of chorioamnionitis had higher temperatures (Table $2, p=0.011$ ) and higher white blood cell counts (Table 2, $p=0.045$ ) than did mothers without chorioamnionitis. Infants with chorioamnionitis had significantly higher immature-to-total neutrophil ratios than did infants without chorioamnionitis (Table 2, $p=0.021$ ). Infants with fetal vessel inflammation had higher I/T ratios than infants without fetal vessel inflammation (median 0.33 versus $0.06, p=0.002$ ). All fifty-five infants had a negative blood culture upon admission to the neonatal intensive care unit.

\section{Cytokines}

Relationship between chorioamnionitis and cytokine concentrations. Cord blood collection from 36 placentas (17 with chorioamnionitis) yielded sufficient plasma for IL- 6 and IL- $1 \beta$ analysis. TNF- $\alpha$ assays were performed for 34 of these. IL-6 was detectable in all plasma samples. The median concentration of IL-6 was significantly higher in infants with than without chorioamnionitis (Fig. 1, $p<0.001$ ). Cord blood IL-6 concentrations were $>25 \mathrm{pg} / \mathrm{mL}$ for $15 / 17$ infants with cho-

Table 1. Infant characteristics

\begin{tabular}{lccc}
\hline & $\begin{array}{c}\text { Chorio } \\
(n=22)\end{array}$ & $\begin{array}{c}\text { No chorio } \\
(n=33)\end{array}$ & $p$ Value \\
\hline Gestational age (wk) & $28.8 \pm 0.5$ & $29.2 \pm 0.4$ & $\mathrm{NS}$ \\
Birth weight (g) & $1286 \pm 78$ & $1305 \pm 66$ & $\mathrm{NS}$ \\
Gender (female) & $55 \%$ & $28 \%$ & $\mathrm{NS}$ \\
Race (non-Caucasian) & $36 \%$ & $20 \%$ & $\mathrm{NS}$ \\
Ventilated at 3 $\pm 1 \mathrm{~h}[n(\%)]$ & $14(64)$ & $18(55)$ & $\mathrm{NS}$ \\
$\mathrm{FIO}_{2}$ & $0.42 \pm 0.19$ & $0.44 \pm 0.21$ & $\mathrm{NS}$ \\
$\mathrm{O}_{2}$ saturation $(\%)$ & $97 \pm 2$ & $97 \pm 3$ & $\mathrm{NS}$ \\
$\mathrm{pH}$ & $7.34 \pm 0.06$ & $7.33 \pm 0.05$ & $\mathrm{NS}$ \\
$\mathrm{PCO}_{2}(\mathrm{~mm} \mathrm{Hg})$ & $46 \pm 6$ & $45 \pm 6$ & $\mathrm{NS}$ \\
\hline
\end{tabular}

Chorio, Chorioamnionitis on pathologic examination of the placenta.

Values shown are mean \pm SD unless noted. 
Table 2. Risk factors and laboratory signs of infection

\begin{tabular}{|c|c|c|c|}
\hline & $\begin{array}{l}\text { Chorio } \\
(n=22)\end{array}$ & $\begin{array}{l}\text { No chorio } \\
(n=33)\end{array}$ & $p$ Value \\
\hline $\begin{array}{l}\text { Clinical suspicion of chorioamnionitis } \\
{[n(\%)]}\end{array}$ & $3(14)$ & $4(12)$ & NS \\
\hline Maternal temperature $\left({ }^{\circ} \mathrm{C}\right)$ & $37.4 \pm 0.7$ & $36.9 \pm 0.6$ & 0.011 \\
\hline $\begin{array}{l}\text { Maternal white blood cell count }(\times \\
\left.10^{6} / \mathrm{L}\right)\end{array}$ & $15.3 \pm 3.1$ & $12.7 \pm 5.0$ & 0.045 \\
\hline $\begin{array}{l}\text { Newborn white blood cell count }(\times \\
\left.10^{6} / \mathrm{L}\right)\end{array}$ & $11.9 \pm 6.3$ & $7.9 \pm 3.6$ & 0.010 \\
\hline $\begin{array}{l}\text { Newborn immature/total white blood } \\
\text { cell ratio }\end{array}$ & $0.26 \pm 0.27$ & $0.10 \pm 0.16$ & 0.021 \\
\hline
\end{tabular}

Chorio, Chorioamnionitis on pathologic examination of the placenta. Values shown are mean \pm SD unless noted.

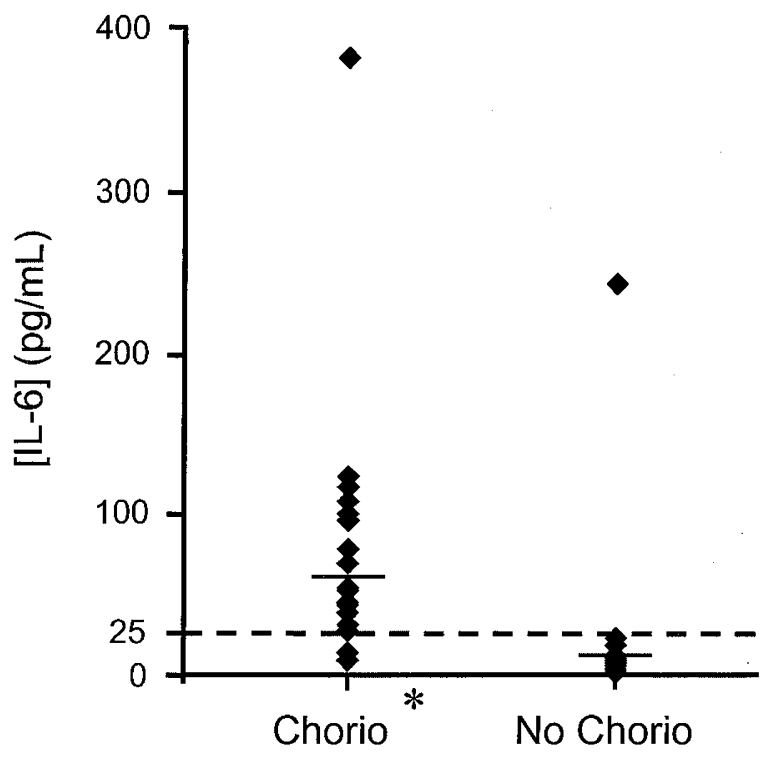

Figure 1. Cord blood IL-6 concentrations grouped by the presence or absence of chorioamnionitis (Chorio). Median IL-6 was higher in infants with chorioamnionitis than without chorioamnionitis (No Chorio) (54.7 vs $10.2 \mathrm{pg} / \mathrm{mL},{ }^{*} p$ $<0.001)$. Fifteen of the 17 infants with chorioamnionitis had an IL-6 $>25$ $\mathrm{pg} / \mathrm{mL}$. Cord blood IL-6 was $<25 \mathrm{pg} / \mathrm{mL}$ in $18 / 19$ infants without chorioamnionitis. One infant grouped as No Chorio had an IL-6 of $244 \mathrm{pg} / \mathrm{mL}$ and was born to a mother with clinical evidence of chorioamnionitis that was not confirmed by placental histology.

rioamnionitis, and were $<25 \mathrm{pg} / \mathrm{mL}$ for $18 / 19$ infants without chorioamnionitis. Thus, a cord blood IL- $6>25 \mathrm{pg} / \mathrm{mL}$ predicted the presence of chorioamnionitis with a sensitivity of $88.0 \%$, a specificity of $94.7 \%$, a positive predictive value of $93.8 \%$, and a negative predictive value of $90.0 \%$.

IL-1 $\beta$ was detectable in cord blood from five infants, all of whom had chorioamnionitis (chorioamnionitis versus no chorioamnionitis, $p=0.035$ ), and all of whom had an IL-6 concentration of $>25 \mathrm{pg} / \mathrm{mL}$ (median $79 \mathrm{pg} / \mathrm{mL}$, range $41-383$ $\mathrm{pg} / \mathrm{mL}$ ). TNF- $\alpha$ was detectable in the cord blood of 11 infants, 8 of whom had chorioamnionitis. Neither the ability to detect TNF- $\alpha$ nor the TNF- $\alpha$ concentration could distinguish infants with chorioamnionitis from infants without chorioamnionitis.

Relationship between fetal vessel inflammation and cytokine concentrations. Fetal vessel inflammation was present in the placentas from 7 of the 36 infants for whom cord blood analysis was performed. Infants with fetal vessel inflammation had higher concentrations of IL-6 [median (range): 69 (41-
383) versus 13 (1-244) $\mathrm{pg} / \mathrm{mL}, p=0.005]$ and were more likely to have detectable concentrations of IL- $1 \beta$ ( $\mathrm{n}=3 / 7$ versus $2 / 29, p=0.04$ ) than were infants without fetal vessel inflammation. However, TNF- $\alpha$ was detected at similar frequencies in infants with as without fetal vessel inflammation.

\section{Hemodynamic Variables}

Relationship between chorioamnionitis and hemodynamic variables. Heart rate was higher in infants with chorioamnionitis than in infants without chorioamnionitis $(153 \pm 13$ versus $145 \pm 13$, respectively; $p=0.027$ ). Mean and diastolic blood pressures (Fig. 2, $p=0.026$ and $p=0.019$, respectively) were lower in infants with than without chorioamnionitis. Systolic blood pressure and pulse pressure did not differ between groups. There was no difference in frequency or direction of ductus arteriosus shunt between infants with chorioamnionitis (absent: $n=1$; left-to-right: $n=21$ ) and without chorioamnionitis (absent: $n=1$; bidirectional: $n=4$; left-to-right: $n=$ 28). Right and left middle cerebral artery systolic, mean, and end-diastolic blood flow velocities, cerebral relative vascular resistance, right and left ventricular cardiac outputs, stroke volume, and fractional shortening did not differ between infants with or without chorioamnionitis.

Relationship between fetal vessel inflammation and hemodynamic variables. Infants with fetal vessel inflammation had significantly higher right ventricular cardiac output than infants without fetal vessel inflammation (Fig. 3, $p=0.028$ ). No other differences in cardiac function, blood pressure, or cerebral Doppler indices were found in this subgroup.

Relationship between cytokine concentrations and hemodynamic variables. IL- 6 concentrations correlated inversely with systolic $\left(R^{2}=0.17, p<0.01\right)$, mean (Fig. $4 ; R^{2}=0.21$, $p<0.01)$, and diastolic $\left(R^{2}=0.22, p<0.01\right)$ blood pressures. IL-6 concentration did not correlate with middle cerebral artery peak systolic, mean, or end-diastolic blood flow velocities, cerebral relative vascular resistance, or with any measure of cardiac function. Furthermore, IL- $1 \beta$ and TNF- $\alpha$ concentrations did not correlate with any measured hemodynamic variable.

Relationship among nonspecific markers of inflammation, cytokine concentrations, and hemodynamic variables. New-

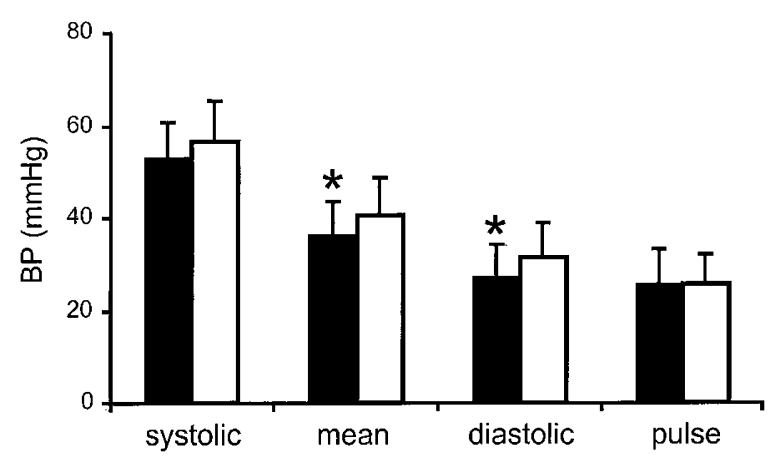

Figure 2. Blood pressure $(B P)$ in infants with (solid bars) and without (open bars) chorioamnionitis. Mean and diastolic blood pressures were lower in infants with chorioamnionitis $(* p<0.05)$. Systolic blood pressure and pulse pressure did not differ. 


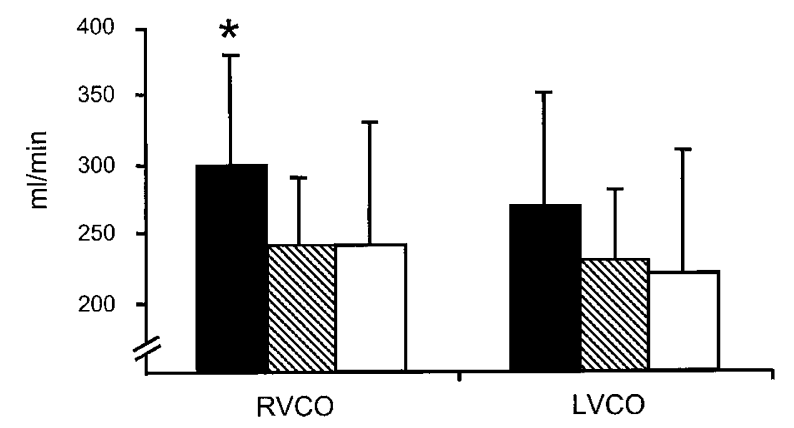

Figure 3. Right $(R V C O)$ and left ventricular cardiac output ( $L V C O)$ of infants with chorioamnionitis in the presence (solid bars, mean $\pm \mathrm{SD}$ ) or absence (shaded bars) of fetal vessel inflammation and in infants without chorioamnionitis (open bars). RVCO was higher in infants with fetal vessel inflammation $\left({ }^{*} p=0.028\right)$ than without. LVCO did not differ among groups.

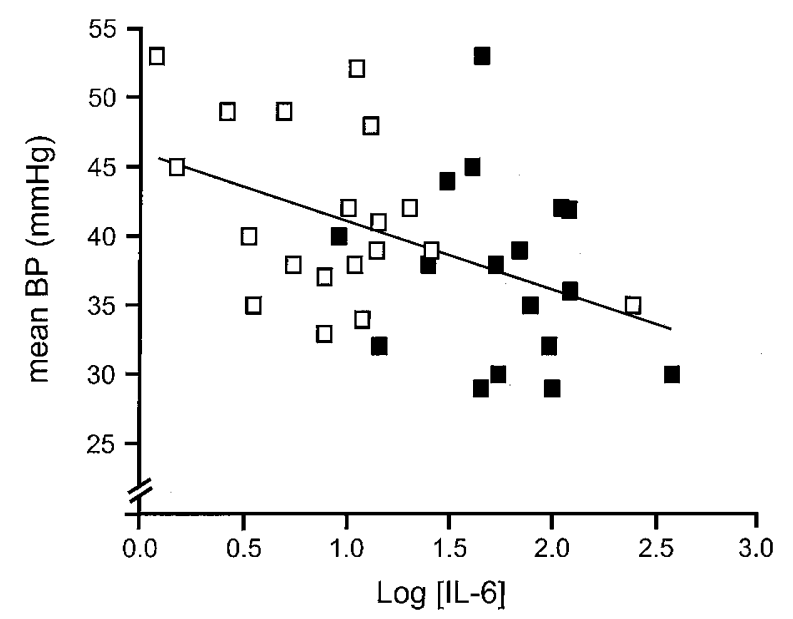

Figure 4. Relationship between mean arterial blood pressure $(B P)$ and IL-6 concentration. Mean BP was inversely correlated $\left(R^{2}=0.21, p<0.01\right)$ with Log IL-6. Solid square $=$ chorioamnionitis; open square $=$ no chorioamnionitis.

born $\mathrm{I} / \mathrm{T}$ ratio correlated positively with cord blood concentrations of IL-6 $\left(R^{2}=0.37, p<0.001\right)$, IL-1 $\beta\left(R^{2}=0.28, p=\right.$ $0.001)$, and TNF- $\alpha\left(R^{2}=0.22, p=0.005\right)$ and inversely with systolic $\left(R^{2}=0.12, p=0.009\right)$, mean $\left(R^{2}=0.12, p=0.009\right)$, and diastolic $\left(R^{2}=0.13, p=0.008\right)$ blood pressures. Newborns whose I/T ratio equaled or exceeded 0.4 had decreased left ventricular fractional shortening ( $32.0 \%$ versus $38.2 \%, p=$ $0.005)$. Left ventricular fractional shortening was also decreased in infants born to febrile mothers $(29.8 \%$ versus $38.2 \%, p=0.001$ ).

Stepwise multiple linear regression was performed on factors that might influence systemic blood pressure including gestational age, number of doses of antenatal steroid received, $\mathrm{pH}$, hematocrit, and cytokine concentrations. Whereas gestational age and IL- 6 concentration were independent predictors of systolic blood pressure, IL-6 concentration was the only significant predictor of mean and diastolic blood pressures.

\section{Cranial Ultrasound Findings}

Five infants were found to have IVH of grade II $(n=4)$ or III $(n=1)$. No infant experienced a parenchymal hemorrhage [Papile grade IV IVH (29)]. Grades II-III IVH occurred more often $(p=0.002)$ in infants whose placentas had evidence of fetal vessel inflammation $(n=4 / 11)$ than in infants who had chorioamnionitis without fetal vessel inflammation $(n=0 / 11)$ or who did not have chorioamnionitis $(n=1 / 33)$.

Cystic PVL was observed on the initial ultrasound of two infants and on the d 14 ultrasound of a third. Ventriculomegaly was present in six infants on d 14, including both infants with initial PVL. The incidences of any white matter injury (PVL and/or ventriculomegaly) were similar between infants with and without chorioamnionitis, and between infants with and without fetal vessel inflammation. Ventriculomegaly was observed more often among infants whose $\mathrm{I} / \mathrm{T}$ ratio was elevated ( $n=3 / 6$ infants with $\mathrm{I} / \mathrm{T} \geq 0.4$ versus $3 / 49$ with $\mathrm{I} / \mathrm{T}$ ratio $<0.4$, $p=0.013)$.

Cord blood was available for two of the five infants with IVH and for all three infants with PVL. All infants with either IVH or PVL for whom cytokine analysis was performed had an IL-6 $>25 \mathrm{pg} / \mathrm{mL}$ [median (range): 36 (27-62) pg/mL]. However, statistical analysis could not be performed because of the small sample size.

No relationships were observed between middle cerebral artery blood flow velocities and cranial ultrasound findings. In regression models to determine the influence of gestational age, left ventricular fractional shortening, mean arterial blood pressure, and elevated newborn I/T ratio on the risk of IVH, PVL or ventriculomegaly, elevated I/T ratio was the strongest predictor of all three brain lesions.

\section{DISCUSSION}

This study was designed to explore the relationship between chorioamnionitis, cord blood cytokine concentrations, and noninvasive indices of hemodynamic function in premature infants at risk for brain injury. Our findings support the hypothesis that chorioamnionitis and elevated plasma concentrations of the proinflammatory cytokines are associated with hemodynamic disturbances among preterm infants.

We found the following:

1. Chorioamnionitis was associated with increased IL-6 and IL- $1 \beta$ concentrations in cord blood.

2. Chorioamnionitis was associated with increased newborn heart rate and with decreased mean and diastolic blood pressures.

3. Cord blood IL-6 concentration correlated inversely with newborn systolic, mean, and diastolic blood pressures.

4. Infants with fetal vessel inflammation had higher IL-6 concentrations, were more likely to have elevated IL- $1 \beta$, and displayed increased right ventricular cardiac output when compared with infants without fetal vessel inflammation.

Our findings are consistent with those of Yoon et al., who have described elevated IL-6 concentrations in cord blood of infants whose placentas manifest chorioamnionitis (16) and funisitis (30). We found that a cord blood IL-6 concentration above $25 \mathrm{pg} / \mathrm{mL}$ was a sensitive and specific predictor of chorioamnionitis. IL-1 $\beta$ was detected infrequently, but was always associated with both inflammatory changes in the placenta and an IL-6 concentration $>25 \mathrm{pg} / \mathrm{mL}$. TNF- $\alpha$ was detected sporadically and was not associated with chorioam- 
nionitis. It is possible that IL- $1 \beta$ and TNF- $\alpha$ concentrations were below the limits of our assay sensitivity for many infants, or that breakdown of protein occurred during processing and sample storage. More likely, IL-6 was most consistently assayable because peak concentrations of IL-6 are reached later and last longer than those of the other proinflammatory cytokines $(31,32)$.

The correlation between cord blood cytokine concentrations and altered hemodynamic function in premature newborns is novel. Although no infant in our study had either a positive blood culture or a diagnosis of septic shock, we were able to demonstrate a strong inverse relationship between IL-6 concentration and blood pressure. The decreased blood pressure and increased cardiac output in infants with placental inflammation suggest that systemic vascular resistance was lower in infants born after chorioamnionitis. When the ductus arteriosus is closed, relative vascular resistance can be calculated as systemic blood pressure divided by left ventricular cardiac output. However, when shunting through the ductus arteriosus is present, left ventricular cardiac output overestimates systemic flow, precluding such calculation. We could not analyze the effects of chorioamnionitis on systemic relative vascular resistance because only two infants had a closed ductus arteriosus at the time of evaluation (2-4 $\mathrm{h}$ after birth).

Cytokines have both direct and indirect effects on vascular smooth muscle tone that can explain the mechanism of cytokine-associated hypotension. TNF- $\alpha$ acts directly on vascular smooth muscle, producing smooth muscle relaxation and hypotension (33). TNF- $\alpha$ and IL-1 $\beta$ act indirectly by increasing vascular endothelial production of prostacyclin (33) and nitric oxide $(34,35)$. Although IL-6 has been associated with hypotension in adults (36), our review of the literature did not support a mechanistic role for IL-6 in this process. Because IL- 6 is induced by and has a longer half-life than IL- $1 \beta$ and TNF- $\alpha(31,32)$, the relationship between IL- 6 and hypotension may represent a temporal, rather than causal, association.

We were unable to demonstrate changes in cerebral blood flow velocities in infants born after chorioamnionitis. Infants with and without chorioamnionitis did not differ with respect to physiologic variables that have been shown to alter cerebral perfusion or disrupt cerebral autoregulation, including acidosis (37) and hypoxia (38). It is possible that global cerebral blood flow was indeed affected by the presence of chorioamnionitis, but that this change could not be detected by Doppler blood flow velocities measured at one point in time. Continuous rather than intermittent monitoring of cerebral blood flow velocities might have revealed either episodic decreases in cerebral perfusion, loss of cerebral autoregulation $(2,39)$ or abnormalities in the normal pattern of maturational change of middle cerebral artery blood flow velocity (40) among the infants born after chorioamnionitis.

Despite the novel findings of this study, we had anticipated finding greater differences between the hemodynamic profiles of infants with and without chorioamnionitis. The inability to detect such differences may be related to the relative stability of our subject population or the limited period of observation. Because our study design required prenatal consent for the collection of specimens at delivery, we may have missed maternal-fetal pairs with the highest grades of chorioamnionitis who would tend to deliver shortly after presentation and before consent could be obtained. The physiologic similarity (Table 1) between infants born to mothers with and without chorioamnionitis supports our premise that the combined cohort represented a relatively healthy subset of the low birth weight population. Furthermore, all study infants had received antenatal steroids. Although we found no differences in hemodynamic measures among infants who received single versus multiple doses of antenatal steroid [current study (data not shown) and (40)], it remains possible that the glucocorticoid lessens hemodynamic disturbances in the newborn by reducing the production of inflammatory mediators. Additional studies performed on infants born to mothers with acutely symptomatic chorioamnionitis, who did not benefit from the positive effects of antenatal steroid administration on cardiac function $(41,42)$, might better distinguish the hemodynamic effects of chorioamnionitis.

Nonetheless, the findings of this study provide insight into the mechanism of infection-associated brain injury among premature infants. Both hypotension $(1,43)$ and placental inflammation (11) have been reported to increase the risk of IVH among premature infants. We found that the likelihood of IVH was increased only among infants whose placentas manifest fetal vessel inflammation. In these newborns, inflammation of the fetal chorionic stem vessels and umbilical cord may reflect a generalized vasculitis affecting both the systemic and the cerebral blood vessels, thereby increasing the propensity for both hypotension and IVH.

Both hypoxia-ischemia (3) and inflammation (11-13) have been implicated in the pathogenesis of cystic PVL. PVL might develop in the hypotensive premature infant if coincident hypoxia (38) and/or acidosis (37) result in loss of autoregulation and thus reduced cerebral blood flow. Our study confirms that chorioamnionitis is associated with reduced blood pressure among premature infants, providing at least one significant risk factor for brain injury. We could not confirm the previously described (13) relationship between cord blood IL-6 concentrations and cystic PVL. This is likely related to statistical power because the sample size was small and cystic PVL is rare, occurring in $3 \%$ to $8 \%$ of low birth weight infants (12, 44). Furthermore, the cysts characteristic of PVL on ultrasound are transient in nature, and may have been apparent in additional infants with more frequent neuroimaging.

Until cytokine analysis is routinely available, the newborn immature-to-total white blood cell ratio may prove to be a practical screening test for the identification of premature infants at risk for hemodynamic disturbances and brain injury. The I/T ratio is available for all infants as an integral part of routine laboratory evaluation. We found that the I/T ratio correlated positively with cord blood IL-6, IL- $1 \beta$, and TNF- $\alpha$ concentrations and inversely with systolic, mean, and diastolic blood pressures. Infants with $\mathrm{I} / \mathrm{T}$ ratios above 0.4 demonstrated poor cardiac contractility. In regression analysis, an elevated I/T ratio was the strongest predictor of both IVH and ventriculomegaly. This is noteworthy because ventriculomegaly may indicate diffuse white matter injury (45) and may be as important as cystic PVL in predicting subsequent handicap $(45,46)$. 
In conclusion, we found that histologic evidence of chorioamnionitis was associated with elevated cord blood IL-6 and IL-1 $\beta$ concentrations, increased newborn heart rate, and decreased blood pressure. Cord blood IL- 6 concentration was the most sensitive and specific plasma marker of chorioamnionitis, and the strongest predictor of newborn blood pressure. Nonspecific indicators of infection, including maternal fever and elevated newborn immature-to-total white blood cell count, were associated with decreased left ventricular function. Thus, our study supports the hypothesis that hemodynamic alterations accompany the inflammatory response syndrome of newborn premature infants when pregnancy is complicated by chorioamnionitis. We speculate that systemic cytokines produce hypotension in these very low birth weight infants. We further speculate that the risk of brain injury is increased in premature infants born after chorioamnionitis when the systemic vasculitis is accompanied by cerebrovascular inflammation.

\section{REFERENCES}

1. Bada H, Korones S, Perry E, Arheart K, Ray J, Pourcyrous M, Magill H, Runyan W, Somes G, Clark F, Tullis K 1990 Mean arterial blood pressure changes in premature infants and those at risk for intraventricular hemorrhage. J Pediatr 117:607-614

2. Perlman J, McMenamin J, Volpe J 1983 Fluctuating cerebral blood flow velocity in respiratory-distress syndrome. Relation to the development of intraventricular hemorrhage. N Engl J Med 309:204-209

3. Volpe J 1995 Neurology of the Newborn. WB Saunders, Philadelphia, pp 296-299

4. Kinney H, Back S 1998 Human oligodendroglial development: relationship to periventricular leukomalacia. Semin Pediatr Neurol 5:180-189

5. DeReuk J, Chattha A, Richardson E 1972 Pathogenesis and evolution of periventricular leukomalacia in infancy. Arch Neurol 27:229-236

6. Takashima S, Tanaka K 1978 Development of cerebrovascular architecture and its relationship to periventricular leukomalacia. Arch Neurol 35:11-16

7. Young R, Hernandez M, Yagel S 1982 Selective reduction of blood flow to white matter during hypotension in newborn dogs: a possible mechanism of periventricular leukomalacia. Ann Neurol 12:445-448

8. Adinolfi M 1993 Infectious diseases in pregnancy, cytokines, and neurological impairment: an hypothesis. Dev Med Child Neurol 35:549-553

9. Kuban K 1998 White-matter disease of prematurity, periventricular leukomalacia, and ischemic lesions. Dev Med Child Neurol 40:571-573

10. Leviton A 1993 Preterm birth and cerebral palsy: is tumor necrosis factor the missing link? Dev Med Child Neurol 35:553-558

11. Dammann O, Leviton A 1997 Maternal intrauterine infection, cytokines, and brain damage in the preterm newborn. Pediatr Res 42:1-8

12. Perlman J, Risser R, Broyles S 1996 Bilateral cystic periventricular leukomalacia in the premature infant: associated risk factors. Pediatrics 97:822-827

13. Yoon B, Jun J, Romero R, Park K, Gomez R, Choi J-H, Kim I-O 1997 Amniotic fluid inflammatory cytokines (interleukin-6, interleukin-1 beta, and tumor necrosis factoralpha), neonatal brain white matter lesions, and cerebral palsy. Am J Obstet Gynecol $177: 19-26$

14. Grether J, Nelson K 1997 Maternal infection and cerebral palsy in infants of normal birth weight. JAMA 278:207-211

15. Hillier S, Witkin S, Krohn M, Watts D, Kiviat N, Eschenbach D 1993 The relationship of amniotic fluid cytokines and preterm delivery, amniotic fluid infection, histologic chorioamnionitis, and choramnion infection. Obstet Gynecol 81:841-948

16. Yoon B, Romero R, Yang S, Jun J, Kim I-M, Choi J-H, Syn H 1996 Interleukin-6 concentrations in umbilical cord plasma are elevated in neonates with white matter lesions associated with periventricular leukomalacia. Am J Obstet Gynecol 174:1433-1440

17. Yoon B, Romero R, Kim C, Jun J, Gomez R, Choi J-H, Syn H 1995 Amniotic fluid interleukin-6: a sensitive test for antenatal diagnosis of acute inflammatory lesions of preterm placenta and prediction of perinatal morbidity. Am J Obstet Gynecol 172:960-970
18. Gomez R, Romero R, Ghezzi F, Yoon B, Mazor M, Berry S 1998 The fetal inflammatory response syndrome. Am J Obstet Gynecol 179:194-202

19. Vassalli P 1992 The pathophysiology of tumor necrosis factors. Annu Rev Immunol 10:411-452

20. Dinarello C 1996 Cytokines as mediators in the pathogenesis of septic shock. Curr Top Microbiol Immunol 216:133-165

21. Shibata M, Parfenova H, Zuckerman S, Leffler C 1996 Tumor necrosis factor-alpha induces pial arteriolar dilation in newborn pigs. Brain Res Bull 39:241-247

22. Tureen J 1995 Effect of recombinant human tumor necrosis factor-alpha on cerebral oxygen uptake, cerebral fluid lactate, and cerebral blood flow in the rabbit: role of nitric oxide. J Clin Invest 95:1086-1091

23. de Vries H, Blom-Roosemalen M, van Oosten M, de Boer A, van Berkel T, Breimer D, Kuiper J 1996 The influence of cytokines on the integrity of the blood-brain barrier in vitro. J Neuroimmunol 64:37-43

24. Mark K, Miller D 1999 Increased permeability of primary cultured brain microvessel endothelial cell monolayers following TNF- $\alpha$ exposure. Life Sci 64:1941-1953

25. Young G 1995 Neurologic complications of systemic critical illness. Neurol Clin 13:645-658

26. Park M, Menard S 1981 Accuracy of blood pressure measurement by Dynamap monitor in infants and children. Pediatrics 19:901-914

27. Greig P, Ernest J, Teot L, Erikson M, Talley R 1993 Amniotic fluid interleukin-6 levels correlate with histologic chorioamnionitis and amniotic fluid cultures in patients in premature labor with intact membranes. Am J Obstet Gynecol 169:10351044

28. Yanowitz T, Yao A, Pettigrew K, Werner J, Oh W, Stonestreet B 1998 Effects of prophylactic low-dose indomethacin on hemodynamics in very low birth weight infants. J Pediatr 132:28-34

29. Papile L-A, Burstein J, Burstein R, Koffler H 1978 Incidence and evolution of subependymal and intraventricular hemorrhage: a study of infants with birth weights less than 1,500 gm. J Pediatr 92:529-534

30. Yoon B, Romero R, Park J, Kim M, Oh S-Y, Kim C, Jun J 2000 The relationship among inflammatory lesions of the umbilical cord (funisitis), umbilical cord plasma interleukin 6 concentration, amniotic fluid infection, and neonatal sepsis. Am J Obstet Gynecol 183:1124-1129

31. van Deventer S, Buller H, ten Cate J, Aarden L, Hack C, Sturk A 1990 Experimental endotoxemia in humans: analysis of cytokine release and coagulation, fibrinolytic, and complement pathways. Blood 76:2520-2526

32. Creasey A, Stevens P, Kenney J, Allison A, Warren K, Catlett R, Hinshaw L, Taylor F 1991 Endotoxin and cytokine profile in plasma of baboons challenged with lethal and sublethal Escherichia coli. Circ Shock 33:84-91

33. Abbas A, Lichtman A, Pober J 1994 Cellular and Molecular Immunology, 2nd Ed. WB Saunders, Philadelphia, pp 245-251

34. Kuhl S, Rosen H 1998 Nitric oxide and septic shock. West J Med 168:176-181

35. Schoonover L, Steward A, Clifton G 2000 Hemodynamic and cardiovascular effects of nitric oxide modulation in the therapy of septic shock. Pharmacotherapy 20:11841197

36. Pinsky M, Vincent J-L, Deviere J, Alegre M, Kahn R, Dupont E 1993 Serum cytokine levels in human septic shock: relation to multi-system organ failure and mortality. Chest 103:565-575

37. Ong B, Greengrass R, Bose D, Gregory G, Palahniuk R 1986 Acidemia impairs autoregulation of cerebral blood flow in newborn lambs. Can J Anaesth 33:5-9

38. Lou H 1994 Hypoxic-hemodynamic pathogenesis of brain lesions in the newborn. Brain Dev 16:423-431

39. Tsuji M, Saul P, duPlessis A, Eichenwald E, Sobh J, Crocker R, Volpe J 2000 Cerebral intravascular oxygenation correlates with mean arterial pressure in critically ill premature infants. Pediatrics 106:625-632

40. Yanowitz T, Yao A, Pettigrew K, Werner J, Oh W, Stonestreet B 1999 Postnatal hemodynamic changes in very-low- birthweight infants. J Appl Physiol 87:370-380

41. Padbury J, Polk D, Ervin M, Berry L, Ikegami M, Jobe A 1995 Postnatal cardiovascular and metabolic responses to a single intramuscular dose of betamethasone in fetal sheep born prematurely by cesarean section. Pediatr Res 38:709-715

42. Padbury J, Ervin M, Polk D 1996 Extrapulmonary effects of antenatally administered steroids. J Pediatr 128:167-172

43. Garland J, Buck R, Leviton A 1995 Effect of maternal glucocorticoid exposure on risk of severe intraventricular hemorrhage in surfactant-treated preterm infants. J Pediatr 126:272-279

44. Hoon A 1995 Neuroimaging of the high-risk infant: relationship to outcome. J Perinatol 15:389-94

45. Leviton A, Gilles F 1996 Ventriculomegaly, delayed myelination, white matter hypoplasia, and "periventricular" leukomalacia: how are they related? Pediatr Neurol 15:127-136

46. Garfinkel E, Tejani N, Boxer H, Levinthal C, Atluru V, Tuck S, Vidyasagar S 1988 Infancy and early childhood follow-up of neonates with periventricular or intraventricular hemorrhage or isolated ventricular dilation: a case controlled study. Am J Perinatol 5:214-219 